\title{
Detecting Intralabyrinthine Pressure Increase by Postural Manipulation with Wideband Tympanometry and Distortion Product Otoacoustic Emissions
}

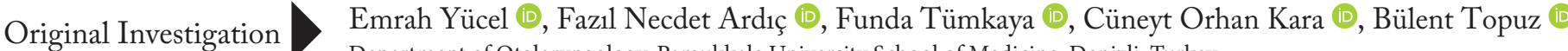 \\ Department of Otolaryngology, Pamukkale University School of Medicine, Denizli, Turkey
}

\begin{abstract}
Objective: Intracranial pressure increase is known to affect inner ear pressure through the cochlear and vestibular aqueducts. This finding forms a good model for inner ear pressure studies. Standard techniques used to detect this pressure increase are neither reliable nor easily repeatable or cheap. Studies with immitancemetry and otoacoustic emissions have been giving hopeful results. This study aims to confirm the results in the literature with wideband tympanometry and add a new parameter of otoacoustic emissions to inner ear pressure testing.
\end{abstract}

ORCID iDs of the authors: E.Y. 0000-0003-0819-6135; F.N.A. 0000-0003-4230-3141; F.T. 0000-0002-3213-8106; C.O.K. 0000-0003-2219-4283; B.T. 0000-0001-7895-6190.

Cite this article as: Yücel E, Ardic FN, Tümkayo F, Kara CO, Topuz B. Detecting Intralabyrinthine Pressure Increase by Postural Manipulation with Wideband Tympanometry and Distortion Product Otoacoustic Emissions. Turk Arch Otorhinolaryngol 2020; 58(4):203-7.

\section{Corresponding Author:}

Fazıl Necdet Ardiç; fnardic@pau.edu.tr

Received Date: 24.05.2020

Accepted Date: 15.09 .2020

Content of this journal is licensed under a Creative Commons Attribution 4.0 International License. Available online at www.turkarchotolaryngol.net
Methods: Wideband tympanometry (WBT) and distortion product otoacoustic emissions (DPOAE) tests were applied to 40 healthy participants in sitting, supine, and Trendelenburg positions. DPOAE were measured under ambient or peak pressure. Resonance frequency, tympanic peak pressure, 1000, 1500, 2000, 3000,

\section{Introduction}

It is a well-known assumption that some of the inner ear diseases become symptomatic with the pressure change in the inner ear compartment. The most famous example is Ménière's disease. Besides Ménière's disease, viral, autoimmune, traumatic disorders of the inner ear may cause the same problem. Therefore, estimating the inner ear pressure accurately is an important goal in audiology. Up to now, electrocochleography, glycerol test, vestibular evoked myogenic potentials (VEMP), otoacoustic emissions (OAE), tympanic membrane displacement analyzer, three-dimensional fluid-attenuated recovery magnetic resonance imaging (MRI) with intratympanic or intravenous gadolinium were used for this purpose (1-4). The
4000 , and $6000 \mathrm{~Hz}$ frequencies in DPOAE were measured.

Results: The increase in the tympanic peak pressure and the decrease in resonance frequency (RF) due to position change were found statistically significant $(\mathrm{p}<0.01)$. Signal noise ratio $(\mathrm{SNR})$ decrease at $1 \mathrm{kHz}$ frequency and SNR increase at 2,3,6 kHz in the normal protocol, SNR decrease at $1 \mathrm{kHz}$ in the pressurized protocol were found statistically significant $(\mathrm{p}<0.01)$.

Conclusion: RF in WBT and $1 \mathrm{kHz}$ DPOAE SNR parameters were found useful in supporting the diagnosis in pathologies that increase intracranial pressure and inner ear pressure. Future research may ease their widespread use in clinical practice as they are non-invasive and rapidly applicable.

Keywords: Inner ear, resonance frequency analysis, tympanometry, otoacoustic emission, intracranial pressure reliability, repeatability, and convenience of these methods have been studied continuously.

One of the first tests introduced for the inner ear pressure was electrocochleography (ECoG). Increased ratio of summating potential to the compound action potential over $>0.40$ is generally accepted as a significant sign of inner ear hydrops. Hornibrook et al. (1) tested patients with definite Ménière's disease and found that tone burst ECoG, click ECoG and MRI showed positivity in $83 \%, 30 \%$, and $47 \%$ of the patients, respectively. Kahn et al. (2) reported that the sensitivity of VEMP was $43 \%$, while sensitivity and specificity of 3 Tesla (3T) MRI intravenous (IV) contrast were $88 \%$ and $89 \%$, respectively. The tympanic 
membrane analyzer was sensitive to inter-individual differences, ossicular chain and middle ear status, and perforations of tympanic membrane. State-of-the-art MRI devices seem to provide the highest accuracy rate for detecting inner ear pressure increase; however, the technique is expensive, hard to repeat, and requires the use of contrast medium that may have side effects.

We still need simple, reliable, and easily repeatable tests. Some researchers focused on immittance tests for this purpose. When inner ear pressure increases, the stapes footplate moves towards the middle ear and stiffness of the annular ligament increases. This, in turn, increases the tension of the ossicular chain, thereby decreases middle ear compliance. All immittancemetric studies that aimed to measure inner ear pressure were based on this assumption (5). There are publications reporting using traditional $226 \mathrm{~Hz}$ tympanometry or multifrequency tympanometry $(6,7)$. It was reported that $2 \mathrm{kHz}$ admittance graphs in the multifrequency tympanogram was affected most by the change of inner ear fluid mechanics. Multifrequency tympanometry (MFT) was believed to measure the pressure of perilymph in direct contact with stapes footplate. A decrease in resonance frequency $(\mathrm{RF})$ and widening of the conductance tympanogram were also noted (4).

Wideband tympanometry (WBT) is a relatively new method. It is an immittancemetric test method that examines the sound transfer function of the middle ear. It uses click stimulus at a range of frequencies between $226 \mathrm{~Hz}$ to $8000 \mathrm{~Hz}(8,9)$.

Several models have been used in inner ear pressure studies. One of these is the glycerol test. Glycerol is an osmotically active compound which reduces the water content of the inner ear and changes the osmolarity (10). The second method is manipulating the posture. Chapman et al. (11) showed that intracranial pressure changed when the posture changed. In two separate studies, Carlborg et al. $(12,13)$ carried out a series of experiments and reported that when the hydrostatic pressure in the subarachnoid space was manipulated, the pressure in the perilymphatic space was affected immediately when the cochlear aqueduct was open. When the cochlear duct was closed, the transfer time was delayed. They postulated that the endolymphatic sac and duct might play a role in this delayed pressure transfer when the cochlear aqueduct is closed. Arterial pressure has a little role in this mechanism. Hypobaric pressure was also a conditional effect depending on the rate of pressure change, patency of the cochlear aqueduct and the Eustachian tube. In another study,

\section{Main Points}

- Postural change from sitting to Trendelenburg affects the inner ear.

- The possible mechanism is the reflection of intracranial pressure increase via the cochlear aqueduct.

- $\quad \mathrm{RF}$ decreases and tympanic peak pressure increases with the postural change in wideband tympanometry.

- $1 \mathrm{kHz}$ signal noise ratio decreases with the postural change in DPOAE.
OAE was also subject to these experiments. A phase increase and level decrease were found especially at frequencies lower than $2 \mathrm{kHz}$ (14). Preliminary studies also showed that posture change created a shift in hearing threshold, OAE, and middle ear impedance (15).

This study aimed to confirm the results reported in the literature for wideband tympanometry and add a new parameter of otoacoustic emissions to inner ear pressure testing in healthy adults.

\section{Methods}

\section{Subjects}

Forty healthy adult volunteers were included in the study. All volunteers underwent detailed otolaryngologic examination. Those with normal ear examination, normal middle ear pressure with $226 \mathrm{~Hz}$ tympanometry, and a hearing threshold over $15 \mathrm{~dB}$ were included, and those with a history of ear disease, neurologic disorder, ototoxic drug use, and ear or cranial surgery were excluded. Volunteers were informed about the procedure and their consents were obtained in writing. The ethical approval of the study was obtained from the Pamukkale University Ethics Committee (Approval Date: February 26, 2016; Approval Number: 60116787-020/13170).

\section{Intervention}

Each ear of participants was measured individually, hence, a total of 80 ears were studied. All subjects were asked not to speak, cough, yawn, or gulp during the procedure.

WBT and OAE measurements were done in three positions, sitting $(\mathrm{O})$, supine $(\mathrm{S})$, and Trendelenburg $(\mathrm{T})\left(15-20^{\circ} \mathrm{head}\right.$ down position), using Titan clinical tympanometry (Titan Suite software; Interacoustics, Denmark). All tests were done immediately after position change.

WBT was performed by narrow band clicks for every $100 \mathrm{~Hz}$ at frequencies changing between 226 to $8000 \mathrm{~Hz}$ and pressure changing between +200 to $-400 \mathrm{daPa}$. Resonance frequency $(\mathrm{RF})$ at tympanic peak pressures (TPP) and pressure values were noted.

Distortion product OAE (DPOAE) was measured using f1 65 $\mathrm{dB}$ SPL, f2 $55 \mathrm{~dB}$ SPL and f1/f2 ratio 1.22. Each test was done twice at ambient and middle ear peak pressure. DP-Gram of frequencies between $1-6 \mathrm{kHz}$ were recorded. Signal noise ratio (SNR) of 1000, 1500, 2000, 3000, 4000, and $6000 \mathrm{~Hz}$ frequencies were used for comparison.

\section{Statistical Analysis}

SPSS version 12.0 (SPSS Inc.; Chicago, IL, USA) was used for statistical analysis. Continuous variables were noted as mean \pm standard deviation. Categorical variables were shown as numbers and percentages. The Kolmogorov-Smirnov test was used to analyze normal distribution, and as the distributions were not normal, nonparametric tests were used for statistical evaluation. A paired sample t-test was done for parametric vari- 
ables. Friedman Test and Wilcoxon signed-rank tests were used for nonparametric variables. We considered $p$ values $<0.05$ as statistically significant.

\section{Results}

A total of 80 ears of 40 volunteers were tested. Twenty-three (57.5\%) were female and $17(42.5 \%)$ were male. Their mean age was calculated as $28.32 \pm 6$.

The average RF of 80 ears in $\mathrm{O}, \mathrm{S}$, and $\mathrm{T}$ positions were 867.68 $\mathrm{Hz}, 855.99 \mathrm{~Hz}$ and $849.99 \mathrm{~Hz}$, respectively. The decrease in RF was found statistically significant $(\mathrm{p}<0.05)$ (Table 1$)$. The average TPP for $\mathrm{O}, \mathrm{S}, \mathrm{T}$ positions were measured as $-1.95,7.63$, $18.76 \mathrm{daPa}$, respectively. The increase was also found statistically significant $(\mathrm{p}<0.05)($ Table 1$)$. There were no statistical differences between right and left ears in RF and TPP.

DPOAE measurements were done under two pressure conditions: ambient and middle ear peak pressure. SNR decreased under an ambient pressure of $1 \mathrm{kHz}$, but increased at 2, 3, and 6 $\mathrm{kHz}$ with position changes. The changes in SNR, whether decreasing or increasing, at $1,2,3,6 \mathrm{kHz}$ were found statistically significant $(\mathrm{p}<0.05)$. But the differences in SNR values at 1.5 and $4 \mathrm{kHz}$ were not significant ( $p>0.05$ ) (Table 2). The right-left

Table 1. WBT measurements during postural change

\begin{tabular}{l|c|c|c|c}
\hline & Sitting & Supine & Trendelenburg & $p$ \\
\hline $\begin{array}{l}\text { Resonance } \\
\text { frequency }(\mathrm{Hz})\end{array}$ & $867.68 \pm 191.67$ & $855.99 \pm 166.34$ & $849.99 \pm 192.5$ & 0.002 \\
\hline $\begin{array}{l}\text { Tympanic } \\
\text { peak pressure } \\
(\mathrm{daPa})\end{array}$ & $-1.95 \pm 8.51$ & $7.63 \pm 9.69$ & $18.76 \pm 12.77$ & 0.0001 \\
\hline
\end{tabular}

Table 2. DP-Grams under ambient pressure were measured and Signal to Noise Ratios were plotted on the table

\begin{tabular}{l|c|c|c|c}
\hline & Sitting & Supine & Trendelenburg & $p$ \\
\hline $1000 \mathrm{~Hz}$ & $7.07 \pm 6.59$ & $6.35 \pm 5.82$ & $3.04 \pm 6.49$ & $0.0001^{*}$ \\
\hline $1500 \mathrm{~Hz}$ & $16.96 \pm 6.5$ & $17.54 \pm 6.56$ & $16.87 \pm 6.5$ & 0.181 \\
\hline $2000 \mathrm{~Hz}$ & $20.44 \pm 6.43$ & $21.2 \pm 6.57$ & $22.7 \pm 6.32$ & $0.003^{*}$ \\
\hline $3000 \mathrm{~Hz}$ & $25.42 \pm 5.17$ & $26.47 \pm 5.48$ & $27.35 \pm 5.2$ & $0.004^{*}$ \\
\hline $4000 \mathrm{~Hz}$ & $25.71 \pm 6.47$ & $26.76 \pm 5.5$ & $26.06 \pm 7.26$ & 0.359 \\
\hline $6000 \mathrm{~Hz}$ & $29.65 \pm 5.89$ & $30.2 \pm 5.68$ & $31.2 \pm 6.03$ & $0.007^{*}$ \\
\hline
\end{tabular}

Table 3. DP-Grams under peak pressure were measured and signal to noise ratio values were plotted in the table

\begin{tabular}{l|c|c|c|c}
\hline & Sitting & Supine & Trendelenburg & $p$ \\
\hline $1000 \mathrm{~Hz}$ & $7.85 \pm 6.08$ & $6.24 \pm 4.97$ & $4.01 \pm 6.72$ & $0.0001^{*}$ \\
\hline $1500 \mathrm{~Hz}$ & $17.42 \pm 6.18$ & $17.75 \pm 6.17$ & $16.56 \pm 7.28$ & 0.078 \\
\hline $2000 \mathrm{~Hz}$ & $20.9 \pm 6.07$ & $21.62 \pm 6.21$ & $22.41 \pm 6.76$ & 0.056 \\
\hline $3000 \mathrm{~Hz}$ & $26.38 \pm 4.66$ & $26.51 \pm 5.56$ & $26.7 \pm 6.14$ & 0.6 \\
\hline $4000 \mathrm{~Hz}$ & $27.04 \pm 5.68$ & $26.52 \pm 5.12$ & $26.5 \pm 5.93$ & 0.62 \\
\hline $6000 \mathrm{~Hz}$ & $29.84 \pm 5.83$ & $29.67 \pm 5.61$ & $30.86 \pm 5.14$ & 0.465 \\
\hline
\end{tabular}

difference was only significant in $3 \mathrm{kHz}$ in the sitting position. When we evaluated the DPOAE under peak pressure, only a decrease in $1 \mathrm{kHz}$ SNR was found significant (Table 3). When we compared right and left ears, $3 \mathrm{kHz}$ SNR under peak pressure was found statistically different in all positions.

\section{Discussion}

In this study, we investigated the change in middle ear RF, TPP, and pressurized/ambient DPOAE while manipulating the pressure of CSF and inner ear by the postural change. We found that $\mathrm{RF}$ decreased and TPP increased when subjects changed from sitting position to the Trendelenburg position. Immediate measurements also showed that $1 \mathrm{kHz}$ SNR decreased with position change under either ambient or peak pressure conditions. The 2, 3 and $6 \mathrm{kHz}$ SNRs increase, but this increase was significant only under ambient pressure.

Normal RF value has been subject to investigations for a long time. Lutman (16) reported RF as $871 \mathrm{~Hz}$ in 1984. Kaya et al. (17) used MFT to measure RF in normal-hearing adults and found that RF was $1020.8 \mathrm{~Hz}$ in right ears and $978.3 \mathrm{~Hz}$ in left ears. Polat et al. (18) focused on the differences between genders and measured mean RF as $933 \mathrm{~Hz}$ and $992.5 \mathrm{~Hz}$ in males and females, respectively. They found this difference to be statistically significant. Another study that evaluated the gender and age differences reported that there were no statistical differences between genders and age groups except in 0-1-month-old babies. In this group, mean RF was detected as 330.4 in the right ear and $347.6 \mathrm{~Hz}$ in the left ear (19). We found the mean RF as $867.68 \pm 191.67 \mathrm{~Hz}$ in sitting position. In the literature, normal $\mathrm{RF}$ was reported to change between $800-1000 \mathrm{~Hz}$. It was postulated that this wide range was due to the changing structure of the outer and middle ears according to age and hereditary traits (20).

WBT is superior to classic tympanometry in many aspects. But the lack of generally accepted normative values is an important limitation. WBT has been widely studied in middle ear diseases. But its capacity for detecting inner ear disorders has always drawn the attention of investigators. One of the major targets was Ménière's disease. To that end, different models were constructed to simulate inner ear pressure increase. One of these is position change. The assumption supporting the positional test with WBT is based on the interaction between the inner ear pressure and the cerebrospinal fluid (11). It has been postulated that when inner ear pressure increases, the stiffness of annular ligament increases, and elasticity of the ossicles decreases. This change could be measured with different techniques. RF is one of these. But there are conflicting results. Franco-Vidal et al. (21) investigated RF and the width of tympanogram change in MFT in different postural positions. When the position was changed from supine to Trendelenburg, the width increased from $141.7 \mathrm{daPa}$ to $184 \mathrm{daPa}$ and $\mathrm{RF}$ increased from $763.7 \mathrm{~Hz}$ to $795.8 \mathrm{~Hz}$. The change in $\mathrm{RF}$ was found insignificant. In another study, same investigators found that RF decreased, and the width of $2 \mathrm{kHz}$ tympanogram increased in Ménière's disease patients (4). So, they focused on 
$2 \mathrm{kHz}$ tympanogram width and concluded that intracochlear pressure could increase between attacks in Ménière's disease (21). Sato et al. (22) tested patients with enlarged vestibular aqueduct syndrome (EVA). They found an RF decrease in EVA. The increase in endolymphatic volume and area of the third window were proposed mechanisms. Sugasawa et al. (23) compared Ménière's disease patients with normal and found decreased RF in Ménière's disease affected ears. They calculated the sensitivity and specificity as 41.3 and $84.2 \%$ when 875 $\mathrm{Hz}$ frequency was chosen as a cutoff point. The normal values in Sugasawa et al.'s (23) series were $1123 \pm 274.8 \mathrm{~Hz}$, which was higher than ours. Kato et al. (24) also reported a good correlation between significant hydrops in MRI and $2 \mathrm{kHz}$ tympanometry width. They concluded that peak width in MFT was an important indicator of Ménière's disease. They also found a tendency of decreasing RF with hydrops, but without any statistically significant difference (24). Cakir Cetin et al. (25), on the other hand, measured RF, absorbance and $2 \mathrm{kHz}$ peak width during the acute attack of Ménière's disease and did not find any significant differences between the affected ears and the non-affected or control ears despite the tendency for increased RF in their results. We found a significant decrease in RF when position was changed from sitting to Trendelenburg. This finding showed that middle ear stiffness was not the only factor affecting RF change. Darrouzet et al. (5) claimed that $\mathrm{RF}$ was not only affected by the stiffness of the annular ligament, but also by the mechanical resistance of the inner ear.

DPOAE was also one of the proposed tests for inner ear pressure studies. Mom et al. (3) investigated the effects of glycerol and postural change in Ménière's disease with transient $\mathrm{OAE}$ and found that there was a positive phase shift in Ménière's disease patients, whereas this shift was inconsistent in the control group. Avan et al. (26) reported that phase shift in Ménière's disease patients was apparent below $1 \mathrm{kHz}$ and increased with postural change, but not significantly, since Ménière's disease had already created a phase shift, postural change did not much affect the result. They also wrote that in 13 Ménière's disease patients, phase shift became normal in the asymptomatic period after having increased phase shift with the symptoms (26). Mom et al. (27) also evaluated the phase shift in Ménière's disease with postural change and reported that phase shift changed between $-30^{\circ}$ to $+45^{\circ}$ in healthy patients, and between $-80^{\circ}$ to $+145^{\circ}$ in Ménière's disease patients. Most of the studies in the literature worked with phase shift, but we evaluated the SNR from DPOAE and found that $1 \mathrm{kHz}$ SNR decreased with the postural change measured either under ambient or peak pressure.

All the OAE studies agreed on the vulnerability or change in low frequencies. Also, in endolymphatic hydrops, hearing loss occurs starting from low frequencies. Densert et al. (28) proposed that hydrops might distort the basilar membrane and cause an abnormal asymmetry in hair cell conductance. Frank and Kössl (29) claimed that this might be due to the mechanical distortion of the outer hair cells only. We found a significant decrease in $1 \mathrm{kHz}$ SNR under ambient and peak pressure with postural change. It was generally recommended that SNR had to be over $6 \mathrm{~dB}$ for good DPOAE measurement. Avan et al. (26) did not find any significant difference in SNR during their phase shift experiments. But all these hypotheses show that the basilar membrane and the outer hair cells are affected by the pressure increase. So, the increase of noise floor might be another parameter for detecting inner ear pressure.

Studies on measuring the changes in the inner ear pressure with immittancemetric tests and DPOAE also brought about a new approach estimating intracranial pressure. Spinal needles or intraventricular catheters are invasive techniques used for this purpose. Avan et al. (30) and Büki et al. (31) studied this subject on computer models, gerbil model, and human subjects, and reported that stiffness of annular ligament affected mostly $1 \mathrm{kHz}$ in DPOAE phase. Experimental studies on cats showed that intracranial pressure immediately affected perilymphatic pressure when the cochlear aqueduct was open; whereas its transfer to the inner ear was delayed when the cochlear aqueduct was closed (13). The assumption and the results were impressive and gave hope for a new widespread application.

\section{Conclusion}

We found that RF decreased and TPP increased when the position of the subject was changed from sitting to Trendelenburg. We also found that especially $1 \mathrm{kHz}$ SNR in DPOAE decreased with the same position change. The increase in other frequencies was not consistent with middle ear pressure change. The high variability of RF makes it hard to compare the results of different studies. Using right-left difference as a factor might be a more reliable criteria. We recommend the use of positional test with WBT in large cohorts before using it in clinical practice. Also, $1000 \mathrm{~Hz}$ DPOAE SNR is worth investigating in these subjects. Further studies in different patient groups with intracranial pressure increase will also enhance our understanding on the relationship between the cerebrospinal fluid and the inner ear.

Ethics Committee Approval: Ethics committee approval was received for this study from the Pamukkale University Ethics Committee (Approval Date: February 26, 2016; Approval Number: 60116787020/13170).

Informed Consent: Informed consent was obtained from the patients who participated in this study.

Peer-review: Externally peer-reviewed.

Author Contributions: Concept - E.Y., F.N.A., F.T., C.O.K., B.T.; Design - E.Y., F.N.A., F.T., C.O.K., B.T.; Supervision - F.N.A., F.T., C.O.K., B.T.; Resources - F.N.A.; Materials - E.Y., F.N.A., F.T.; Data Collection and/or Processing - E.Y., F.N.A.; Analysis and/or Interpretation - E.Y., F.N.A., F.T., C.O.K., B.T.; Literature Search - E.Y., F.N.A., F.T.; Writing - E.Y., F.N.A., C.O.K., B.T.; Critical Reviews F.N.A., F.T., C.O.K., B.T.

Conflict of Interest: The authors have no conflicts of interest to declare.

Financial Disclosure: The authors declared that this study was supported by Pamukkale University Research Fund (Number: 2016TIPF014). 


\section{References}

1. Hornibrook J, Flook E, Greig S, Babbage M, Goh T, Coates M, et al. MRI inner ear imaging and tone burst electrocochleography in the diagnosis of Ménière's disease. Otol Neurotol 2015; 36: 110914.

2. Kahn L, Hautefort C, Guichard JP, Toupet M, Jourdaine C, Vitaux $\mathrm{H}$, et al. Relationship between video head impulse test, ocular and cervical vestibular evoked myogenic potentials, and compartmental magnetic resonance imaging classification in Menière's disease. Laryngoscope 2020; 130: E444-E452.

3. Mom T, Gilain L, Avan P. Effects of glycerol intake and body tilt on otoacoustic emissions reflect labyrinthine pressure changes in Menière's disease. Hear Res 2009; 250: 38-45.

4. Franco-Vidal V, Legarlantezec C, Blanchet H, Convert C, Torti F, Darrouzet V. Multifrequency admittancemetry in Ménière's disease: a preliminary study for a new diagnostic test. Otol Neurotol 2005; 26: 723-7.

5. Darrouzet V, Dulon D, Franco-Vidal V. Multifrequency immittancemetry in experimentally induced stapes, round window and cochlear lesions. Audiol Neurootol 2007; 12: 85-100.

6. Bianchedi M, Croce A, Neri G, Moretti A. [Multifrequency tympanometry in Meniere's disease: preliminary results.] Acta Otorhinolaryngol Ital 1996; 16: 1-5.

7. Gersdorff M. [Clinical value of multifrequential tympanometry.] Acta Otorhinolaryngol Belg 1980; 34: 270-8.

8. Shahnaz N, Bork K, Polka L, Longridge N, Bell D, Westerberg BD. Energy reflectance and tympanometry in normal and otosclerotic ears. Ear Hear 2009; 30: 219-33.

9. Shahnaz N, Longridge N, Bell D. Wideband energy reflectance patterns in preoperative and post-operative otosclerotic ears. Int J Audiol 2009; 48: 240-7.

10. Kanoh N, Fukazawa T, Hirono Y, Makimoto K. Effects of glycerol on the inner ear fluid electrolytes of guinea pigs-oral and intravenous administration. Auris Nasus Larynx 1984; 11: 125-38.

11. Chapman PH, Cosman ER, Arnold MA. The relationship between ventricular fluid pressure and body position in normal subjects and subjects with shunts: a telemetric study. Neurosurgery 1990; 26: 181-9.

12. Carlborg BIR, Konrádsson KS, Carlborg AH, Farmer JC Jr, Densert O. Pressure transfer between the perilymph and the cerebrospinal fluid compartments in cats. Am J Otol. 1992; 13: 41-8.

13. Carlborg BIR, Farmer JC. Transmission of cerebrospinal fluid pressure via the cochlear aqueduct and endolymphatic sac. Am J Otolaryngol 1983; 4: 273-82.

14. de Kleine E, Wit HP, Avan P, van Dijk P. The behavior of evoked otoacoustic emissions during and after postural changes. J Acous Soc Am 2001; 110: 973-80.

15. Büki B, Chomicki A, Dordain M, Lemaire JJ, Wit HP, Chazal J, et al. Middle-ear influence on otoacoustic emissions. II: contributions of posture and intracranial pressure. Hear Res 2000; 140: 202-11.
16. Lutman ME. Phasor admittance measurements of the middle ear. I. theoretical approach. Scand Audiol 1984; 13: 253-64.

17. Kaya Ş, Karabulut M, Keseroğlu K, Er S, Dağlı M, Korkmaz H. Sağliklı yetişkinlerde multi-frekans timpanometri ve orta kulak rezonans frekansı: klinik normatif değerler. KBB Forum. 2020; 19: 27-33.

18. Polat Z, Baş B, Hayır D, Bulut E, Ataş A. Wideband tympanometry normative data for Turkish young adult population. J Int Adv Otol 2015; 11: 157-62.

19. Özgür A, Müjdeci B, Terzi S, Özergin Coşkun Z, Yiğit E, Dursun E. Wideband tympanometry normative data for different age groups in Turkish population. J Int Adv Otol 2016; 12: 82-6.

20. Shanks J, Shelton C. Basic principles and clinical application of tympanometry. Otolaryngol Clin North Am 1991; 24: 299-328.

21. Franco-Vidal V, Bonnard D, Bellec O, Thomeer H, Darrouzet V. Effects of body tilt on multifrequency admittance tympanometry. Otol Neurotol 2015; 36: 737-40.

22. Sato E, Nakashima T, Lilly DJ, Fausti SA, Ueda H, Misawa H, et al. Tympanometric findings in patients with enlarged vestibular aqueducts. Laryngoscope 2002; 112: 1642-6.

23. Sugasawa K, Iwasaki S, Fujimoto C, Kinoshita M, Inoue A, Egami $\mathrm{N}$, et al. Diagnostic usefulness of multifrequency tympanometry for Ménière's disease. Audiol Neurotol 2013; 18: 152-60.

24. Kato K, Yoshida T, Teranishi M, Sano R, Otake H, Sone M, et al. Peak width in multifrequency tympanometry and endolymphatic hydrops revealed by magnetic resonance imaging. Otol Neurotol 2012; 33: 912-5.

25. Cakir Cetin A, Gurkan S, Kirkim G, Guneri EA. Wide-band tympanometry results during an acute episode of Ménière's disease. Audiol Neurootol 2019; 24: 231-6.

26. Avan P, Giraudet F, Chauveau B, Gilain L, Mom T. Unstable distortion-product otoacoustic emission phase in Menière's disease. Hear Res 2011; 277: 88-95.

27. Mom T, Montalban A, Bascoul A, Gilain L, Avan P. Acoustic phase shift: objective evidence for intralabyrinthine pressure disturbance in Menière's disease provided by otoacoustic emissions. Eur Ann Otorhinolaryngol, Head Neck Dis 2012; 129: 17-21.

28. Densert B, Densert O, Arlinger S, Sass K, Ödkvist L. Immediate effects of middle ear pressure changes on the electrocochleographic recordings in patients with Menière's disease: a clinical placebo-controlled study. Am J Otol 1997; 18: 726-33.

29. Frank G, Kössl M. The acoustic two-tone distortions $2 \mathrm{f} 1-\mathrm{f} 2$ and $\mathrm{f} 2 \mathrm{f} 1 \mathrm{f}$ and their possible relation to changes in the operating point of the cochlear amplifier. Hear Res 1996; 98: 104-15.

30. Avan P, Büki B, Maat B, Dordain M, Wit HP. Middle ear influence on otoacoustic emissions. I: noninvasive investigation of the human transmission apparatus and comparison with model results. Hear Res 2000; 140: 189-201.

31. Büki B, de Kleine E, Wit HP, Avan P. Detection of intracochlear and intracranial pressure changes with otoacoustic emissions: a gerbil model. Hear Res 2002; 167: 180-91. 